\title{
Nanogenerators and Nanopiezotronics
}

\author{
Zhong Lin (Z.L.) Wang
}

School of Materials Science and Engineering, Georgia Institute of Technology, Atlanta

The future of nanotechnology research is likely to focus on the areas of integrating individual nanodevices into a nanosystem that acts like living specie with sensing, communicating, controlling and responding. A nanosystem requires a nano-power source to make the entire package extremely small and high performance. The goal is to make self-powered nanosystem that can operate wirelessly, independently and sustainably. Harvesting energy from the environment is a choice for powering nanosystems.

Developing wireless nanodevices and nanosystems are of critical importance for sensing, medical science, defense technology and even personal electronics. It is highly desired for wireless devices and even required for implanted biomedical devices to be selfpowered without using battery. It is essential to explore innovative nanotechnologies for converting mechanical energy (such as body movement, muscle stretching), vibration energy (such as acoustic/ultrasonic wave), and hydraulic energy (such as body fluid and blood flow) into electric energy that will be used to power nanodevices without using battery. This is a key step towards self-powered nanosystems. We have demonstrated an innovative approach for converting nano-scale mechanical energy into electric energy by piezoelectric zinc oxide nanowire (NW) arrays. The operation mechanism of the electric generator relies on the unique coupling of piezoelectric and semiconducting properties of $\mathrm{ZnO}$ as well as the gating effect of the Schottky barrier formed between the metal tip and the NW. Based on this mechanism, we have recently developed DC nanogenerator (NG) driven by ultrasonic wave in bio-fluid and textile fibers based NG for harvesting low frequency mechanical energy. Further more, a new field on nanopiezotronics has been developed, which uses piezoelectric-semiconducting coupled property for fabricating novel and unique electronic devices and components. This review gives a systematic description about the fundamental mechanism of the NG, its rationally innovative design for high output-power, and the new electronics that can be built based on a piezoelectric driven semiconducting process. A perspective will be given about the future impact of the technologies.

References

[1] Z.L. Wang "Self-powering nanotech", Scientific American, 298 (2008) 82-87.

[2] Z.L. Wang and J.H. Song Piezoelectric Nanogenerators Based on Zinc Oxide Nanowire Arrays", Science, 312 (2006) 242-246.

[3] X.D. Wang, J.H. Song J. Liu, and Z.L. Wang "Direct current nanogenerator driven by ultrasonic wave", Science, 316 (2007) 102-105.

[4] Y. Qin, X.D. Wang and Z.L. Wang "Microfiber-Nanowire Hybrid Structure for Energy Scavenging", Nature, 451 (2008) 809-813. 
[5] R.S. Yang, Y. Qin, L.M. Dai and Z.L. Wang "Flexible charge-pump for power generation using laterally packaged piezoelectric-wires", Nature Nanotechnology, 4 (2009) 34-39.

[6] Z.L. Wang "Towards self-powered nanosystems: from nanogenerators to nanopiezotronics", Advanced Functional Materials, 18 (2008) 3553-3567.

[7] Top 10 future technologies by New Scientists:

http://www.newscientist.com/article/mg20126921.800-ten-scifi-devices-that-couldsoon-be-in-your-hands.html?full=true

[8] Research supported by DARPA, BES DOE, NSF, NASA and Emory-Georgia Tech CCNE from NIH.

[9] for details visit: http://www.nanoscience.gatech.edu/zlwang/

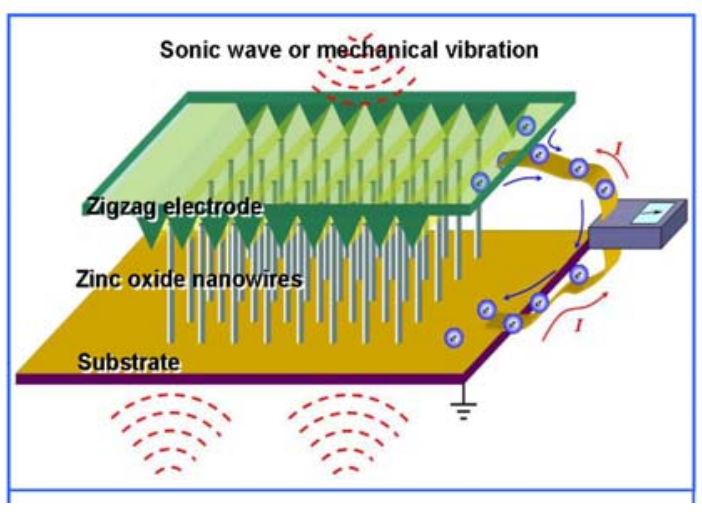

Figure 1. Schematic diagram showing the direct current nanogenerator built using aligned $\mathrm{ZnO}$ nanowire arrays with a zigzag top electrode. The nanogenerator is driven by an external ultrasonic wave or mechanical vibration and the output current is continuous.

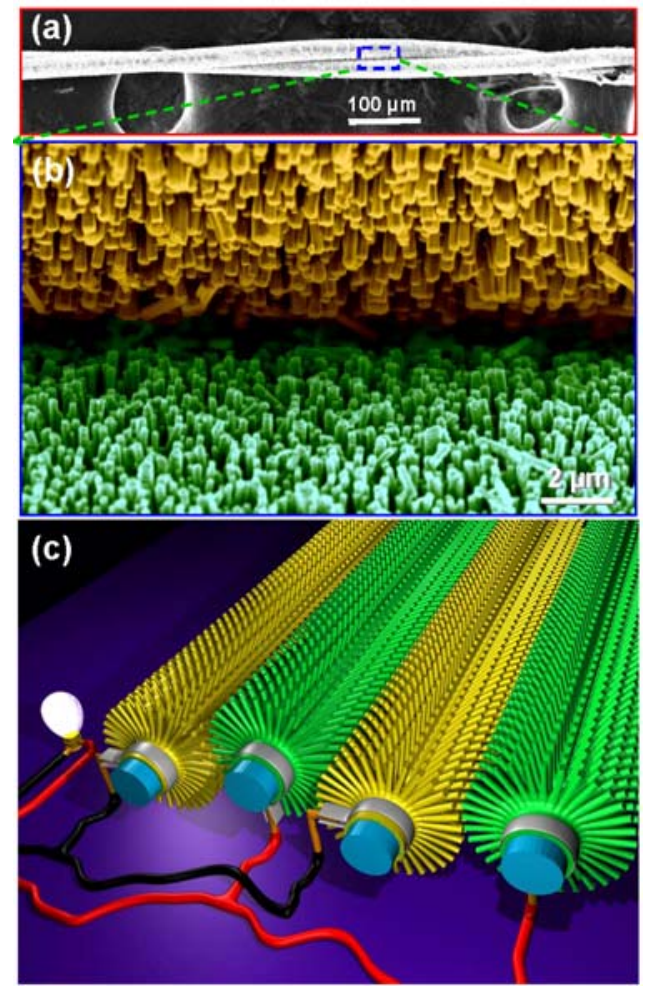

Figure 2. (a) Scanning electron microscopy (SEM) image of two entangled microfibers that were covered radially with piezoelectric $\mathrm{ZnO}$ nanowires, with one of them coated with gold. The relative scrubbing of the two "brushes" generates electricity. (b) A magnified SEM image at the area where the two "brushes" meet teeth-to-teeth, with the top one coated with gold and the bottom one is as-synthesized $\mathrm{ZnO}$ nanowires. (c) A schematic illustration of the microfiber-nanowire hybrid nanogenerator, which is the basis of using fabrics for generating electricity. 